\title{
ANÁLISE PALINOTAXONÔMICA E BIOESTRATIGRÁFICA DA SUBTURMA MONOSACCITES NA BACIA DO PARANÁ, BRASIL. I: PLICATIPOLLENITES LELE 1964 E CRUCISACCITES LELE \& MAITHY 1965
}

\author{
CRISTINA M. FÉLIX, EDUARDO PREMAOR, \\ GUILHERME HERMANY \& PAULO A. SOUZA \\ Departamento de Paleontologia e Estratigrafia, Instituto de Geociências, UFRGS, Cx.P. 15.001, 91.509-900, Porto Alegre, RS. \\ cmfelixrs@yahoo.com.br,premaor@pop.com.br,guilherme.hermany@ufrgs.br,paulo.alves.souza@ufrgs.br
}

\begin{abstract}
RESUMO - O trabalho apresenta a revisão taxonômica dos grãos de pólen monossacados vinculados aos gêneros Plicatipollenites Lele 1964 e Crucisaccites Lele \& Maithy 1964 ocorrentes na bacia do Paraná, onde são representados por seis espécies: P. malabarensis (Potonié \& Sah) Foster 1975, P. gondwanensis (Balme \& Hennelly) Lele 1964, P. trigonalis Lele 1964, P. densus Srivastava 1970, C. latisulcatus Lele \& Maithy 1964 e C. monoletus Maithy 1965. Com base na análise de amostras de localidades inéditas, novas lâminas de material publicado ou proveniente de coleções científicas e no exame das descrições e/ou ilustrações disponíveis na literatura, listas sinonímicas são propostas, modificando algumas amplitudes estratigráficas. Além disso, os principais caracteres morfológicos distintivos para cada espécie são ressaltados, de modo a facilitar sua identificação e seu uso em trabalhos bioestratigráficos e de interpretação paleoambiental.
\end{abstract}

Palavras-chaves: Palinologia, taxonomia, grãos de pólen monossacados, bacia do Paraná.

\begin{abstract}
PALYNOTAXONOMIC AND BIOSTRATIGRAPHIC ANALYSIS OF THE SUBTURMA MONOSACCITES IN THE PARANÁ BASIN, BRAZIL. I. PLICATIPOLLENITES LELE 1964 AND CRUCISACCITES LELE \& MAITHY 1965. This paper presents a taxonomic analysis of the monosaccate pollen grains related to the genera Plicatipollenites Lele 1964 and Crucisaccites Lele \& Maithy 1964 recorded in the Paraná Basin. In this basin, these genera are represented by six species: P. malabarensis (Potonié \& Sah) Foster 1975, P. gondwanensis (Balme \& Hennelly) Lele 1964, P. trigonalis Lele 1964, P. densus Srivastava 1970, C. latisulcatus Lele \& Maithy 1964 and C. monoletus Maithy 1965. Based on analysis of samples from new localities, new slides of published sections or from scientific collections, and on the examination of available papers, synonymic lists are proposed, modifying the ranges of some species. Furthermore, main morphologic characters of distinction between these species are discussed, in order to facilitate the identification and their use in biostratigraphic and paleoenvironmental analysis.
\end{abstract}

Key words: Palynology, taxonomy, monosaccate pollen grains, Paraná Basin.

\section{INTRODUÇÃO}

A seção neopaleozóica da bacia do Paraná apresenta um dos mais importantes registros sedimentares do Gondwana, com notável presença de palinomorfos, principalmente concentrados nas unidades que constituem os grupos Tubarão e Passa Dois, que correspondem à seqüência Gondwana I de Milani \& Zalán (1999) (Figura 1). Adiversidade palinológica é extremamente alta, ensejando diversos trabalhos detalhados de registro e análise taxonômica.

A Subturma Monosaccites Chitaley emend. Potonié \& Kremp 1954 é uma das mais importantes em termos de abundância e número de espécies, agrupando grãos de pólen monossacados intramicrorreticulados, de simetria radial ou bilateral, providos de corpo central com ou sem sistema de pregas de aderência ao saco, com ou sem estrias. Essas feições morfológicas caracterizam os principais gêneros gondvânicos incluídos na subturma, geralmente considerados como representantes das Cordaitales e/ou Coniferales, dentre os quais: Cannanoropollis Potonié \& Sah 1960, Plicatipollenites Lele 1964, Crucisaccites Lele \& Maithy 1964, Potonieisporites Bhardwaj emend. Bharadwaj 1964, Caheniasaccites Bose \& Kar emend. Azcuy \& Di Pasquo 2000, Divarisaccus Venkatachala \& Kar 1966, Striomonosaccites (Bharadwaj) Hart 1965 e Meristocorpus Playford \& Dino 2000.

Alguns destes gêneros foram utilizados como base na delimitação de intervalos bioestratigráficos, formais ou não, para a bacia do Paraná (e.g., Daemon \& Quadros, 1970; Arai, 1980), em virtude de incluírem espécies-guias com ampla distribuição na bacia. Em trabalhos posteriores (e.g., DiasFabrício, 1981; Souza et al., 2003), alguns desses táxons foram revisados, revelando significativas mudanças do ponto de vista 
taxonômico, com implicações bioestratigráficas. No entanto, a quantidade de espécies incluídas na subturma é relativamente ampla, merecendo enfoque particular e detalhado, de modo a estabelecer uma lista mais precisa e taxonomicamente atualizada das espécies ocorrentes na bacia.

Estudos preliminares indicaram a extensão da amplitude estratigráfica de determinadas espécies do grupo, dentre as quais Plicatipollenites trigonalis Lele 1964, P. malabarensis (Potonié \& Sah) Foster 1975 e Cannanoropollis triangularis (Methae) Bose \& Maheshwari 1968 (Souza et al., 2003). Além disso, proposições de novos táxons foram rejeitadas, como, por exemplo, Plicatipollenites paranaensis Cauduro 1970, sinonimizado em $P$. gondwanensis (Balme \& Hennely) Lele 1964 (vide Gutiérrez, 1993 e Premaor et al., 2004).

Nesse contexto, este trabalho constitui-se na primeira comunicação de projeto maior sobre o tema, que envolve a análise taxonômica e bioestratigráfica de todos os espécimes de grãos de pólen monossacados ocorrentes na bacia do Paraná, com base em materiais inéditos e na revisão de trabalhos publicados. Dessa forma, visa à atualização taxonômica e à melhor determinação da amplitude estratigráfica da subturma, a exemplo do que já foi feito para outras bacias do continente sul-americano (e.g., Gutiérrez, 1993; Azcuy \& Di Pasquo, 2000). Os resultados deverão ser aplicados na palinobioestratigrafia, embasando correlações intra e extrabacinais, e nas interpretações paleoambientais e paleoclimáticas.

\section{MATERIAIS E MÉTODOS}

Este trabalho é baseado na análise de amostras de novas localidades, lâminas preparadas de material publicado ou proveniente de coleções científicas, bem como nas descrições e/ou ilustrações disponíveis na literatura para a bacia do Paraná. Adicionalmente, também foram consultados trabalhos inéditos, referentes a dissertações, teses e relatórios internos.

Foram processadas amostras de material inédito e confeccionadas novas lâminas de materiais previamente estudados, procurando-se abranger todo o pacote sedimentar palinologicamente produtivo do Paleozóico Superior da bacia do Paraná. Do Subgrupo Itararé foram selecionados os afloramentos de Mariana Pimentel (RS), Itu (SP), Monte Mor (SP) e Jundiaí (SP) e níveis dos poços IG-01 (Itaporanga, SP), ITIG-85 (Itu, SP) e A-IG-85 (Araçoiaba da Serra, SP); da Formação Rio Bonito, o afloramento de Mariana Pimentel (RS); da Formação Palermo, níveis do poço 2-PN-1-SP (Paranapanema, SP); da Formação Irati, o afloramento de Montividiu (GO) níveis do poço F177 (Minas do Leão, RS); e, um nível da Formação Corumbataí (poço PL07, SP). Todas as lâminas estão depositadas na Palinoteca do Museu de Paleontologia do Departamento de Paleontologia e Estratigrafia do IG/UFRGS.

Visando o entendimento taxonômico adequado dos gêneros e espécies abordados, as descrições e diagnoses originais foram analisadas e comparadas com táxons afins, de forma a promover a confecção de listas sinonímicas atualizadas, com a base nomenclatural para cada táxon (e.g., basiônimo, emendas e combinações) e espécimes registrados da bacia do Paraná. Para efeito de distribuição estratigráfica, foram considerados os trabalhos disponíveis com descrições e/ou ilustrações adequadas para análise, incluindo, quando pertinentes, monografias, dissertações e teses. No caso de um espécime ter sido ilustrado em mais de uma publicação, utilizouse aquela mais antiga, desde que resguardada a confiabilidade da procedência de cada material, a fim de não prejudicar as interpretações sobre sua amplitude.

As descrições de novos materiais foram realizadas sob microscópio óptico em aumentos entre 100 e 1.000 vezes, com documentação fotomicrográfica em câmera digital acoplada ao microscópio Olympus BX 51 do Laboratório de Palinologia do Instituto de Geociências da UFRGS (IGEO/UFRGS). As lâminas do material fotografado estão depositadas nas palinotecas do IGEO/UFRGS (sob código MP-P), Instituto de Geociências da USP (GP4E) e do Instituto Geológico/SMA (IG-P).

\section{PALINOLOGIA SISTEMÁTICA}

Nesta seção são apresentadas as descrições taxonômicas de quatro espécies de Plicatipollenites e duas de Crucisaccites ocorrentes na bacia do Paraná, agrupados conforme esquema parataxonômico proposto por Potonié (1970) e Potonié \& Kremp (1954).

\section{Anteturma VARIEGERMINANTES Potonié 1970 \\ Turma SACCITES Erdtmann 1947}

Subturma MONOSACCITES Chitaley emend.Potonié \& Kremp 1954 Infraturma TRILETESACCITI Leschik 1955

\section{Plicatipollenites Lele 1964}

Punctasaccites Bose \& Kar 1966 (pars.).

Espécie-tipo. Plicatipollenites malabarensis (Potonié \& Sah) Foster 1975.

Afinidade botânica. Gymnospermopsida (Coniferales e Cordaitales) (Bharadwaj, 1957; Potonié, 1967; Clement-

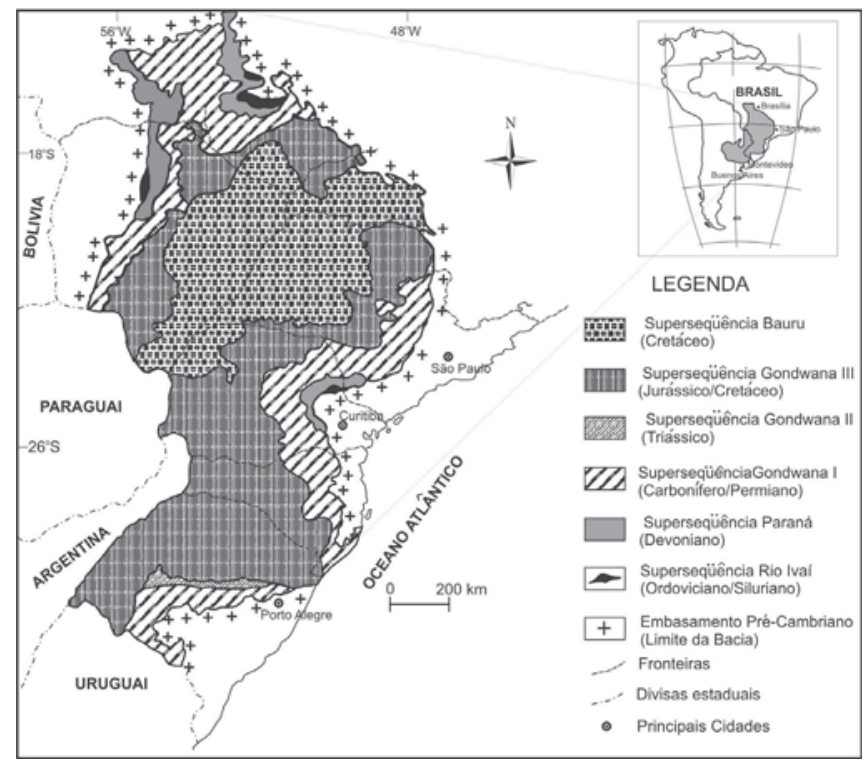

Figura 1. Distribuição das superseqüências estratigráficas da bacia do Paraná (baseado em Milani, 1997; modificado de Souza \& Marques-Toigo, 2003).

Figure 1.Distribution of Paraná Basin stratigraphic supersequences (based on Milani, 1997; modified from Souza \& Marques-Toigo, 2003). 
Westerhoff, 1984).

Aspectos gerais. O gênero Plicatipollenites inclui grãos de pólen monossacados, de simetria radial, com contorno geral variando de triangular, subcircular a circular, saco aderido equatorialmente na face proximal do corpo central e subequatorialmente na face distal, onde é associado a um sistema de pregas; corpo central de contorno variado (triangular, subcircular a circular), nem sempre acompanhando o contorno geral do grão (Lele, 1964, p. 151-153), geralmente com marca trilete visível. Segundo Azcuy \& Gutiérrez (1985, p. 101-102), o sistema de pregas é um caráter diagnóstico do gênero, cuja forma é distintiva na definição de suas espécies, juntamente com os contornos gerais do grão e do corpo central e relação das dimensões entre o corpo central e o saco.

Comparação com outros gêneros. Nuskoisporites Potonié \& Klaus 1954 não apresenta sistema de pregas associado à aderência distal do saco no corpo central, além de que este é envolvido por todo o saco. Barakarites Bharadwaj \& Tiwari 1964 possui "tenuítas" circumpolares na face proximal do corpo central, que lhe confere aspecto bizonado. Para Jansonius \& Hills (1976, ficha $n^{\circ} 2284$ ), o pontoamento do corpo central em Punctasaccites Bose \& Kar 1966 constitui efeito de preservação (erosão), opinião aqui compartilhada, corroborando Gutiérrez (1993, p. 167). Dessa forma, sua espécie tipo, Punctasaccites ovatus Bose \& Kar (1966, est. 2, figs. 2-3, p. 22), é relocada a Plicatipollenites gondwanensis, enquanto Punctasaccites ellipticus (Bose \& Kar 1966, est. II, fig. 4, p. 23) deve ser incluído no gênero Potonieisporites Bharadwaj emend. Bharadwaj 1964, por apresentar simetria bilateral e sistema de pregas associado à raiz distal do saco no corpo central. Varlamoffites Bose \& Kar 1966 possui marca monolete, além de sistema de pregas circumpolar, de modo que o saco envolve maior área do corpo central na face distal do que em Plicatipollenites. Considerando-se a marca monolete como caráter funcional, justifica-se a separação dos dois gêneros. No entanto, faltam informações sobre o número de espécimes tratados por Bose \& Kar (1966) na construção da espécie tipo do gênero, para um melhor julgamento sobre a constância dessa feição e seu estado de preservação. No Brasil, Playford \& Dino (2000, est. 2, fig. 11, p. 96) e Souza et al. (2003, est. 4, fig. 4, p. 62) registraram espécimes semelhantes, com marca monolete, atribuídas a Plicatipollenites sp. cf. P. gondwanensis e Plicatipollenites sp., respectivamente, que se diferenciam de Varlamoffites pela presença de sistema de pregas na região subequatorial do corpo central.

Alguns espécimes registrados para a bacia do Paraná exibem feições diagnósticas que permitem enquadramento taxonômico tanto em $P$. malabarensis quanto em $P$. gondwanensis. Os espécimes ilustrados por Daemon \& Quadros (1970, est. 3, fig. B-P550), Souza (1996, est. 6, fig. 4, p. 92) e Souza et al. (2003, est. 4, fig 5, p. 62) apresentam sistema de pregas aproximadamente poligonal, típico de $P$. gondwanensis, mas ocupando posição próxima à margem do corpo central. Além disso, nestes espécimes, o saco apresenta pouca largura na área além do corpo central, o que é típico de P. malabarensis. Estas feições também são verificadas nos espécimes ilustrados por Lindström (1995, est. IX, fig. 9), da Antártida, e Azcuy \& Di Pasquo (2000, est. 1, fig. 9), da Argentina.

Processos tafonômicos afetam a preservação dos espécimes, podendo promover a fragmentação dos grãos e alterar feições morfológicas diagnósticas, com implicações na identificação taxonômica. Os espécimes ilustrados por Picarelli (1986, est. 3, fig. 5), Piccoli et al. (1991, est. 2, fig. a), Guerra-Sommer et al. (1995a, est. 5, fig. 2) e Souza (1996, est. 6, fig. 4) apresentam deformações, sobretudo, na posição do sistema de pregas, levemente deslocado, e no contorno geral do grão. Estas limitações impedem a distinção entre $P$. malabarensis e $P$. gondwanensis.

Os espécimes ilustrados em Daemon \& Quadros (1970, est. 7, fig. P-402), Kemp (1975, est. 29II, fig. 15), Dellazzana (1976, est. IV, fig. 11 e est.V, fig. 1) e Souza et al. (1993, est. 3, fig. 9), atribuídos, respectivamente, a Nuskoisporites sp., $P$. trigonalis, Cordaitina sp. e P. trigonalis, apresentam contorno aproximadamente subtriangular e ampla largura relativa do saco, podendo ser referidos ao gênero Plicatipollenites, porém sem designação específica.

Plicatipollenites malabarensis (Potonié \& Sah) Foster 1975 (Figuras 2A, B)

Cannanoropollis malabarensis Potonié \& Sah, 1960:128-129 (basiônimo), est. 2, figs. 19-21 e est. 3, fig. 22.

Plicatipollenites indicus Lele, 1964:152-154, est. 1, figs. 610, text-fig. 3a-fe 12 a.

Plicatipollenites paranaensis Cauduro, 1970:14, pars, est. 10, fig. 72.

Plicatipollenites malabarensis (Potonié \& Sah) Foster, 1975:142, est. 5, fig. 1.

Plicatipollenites indicus (auct. non) Lele 1964 in Daemon \& Quadros, 1970:382, est. 3, forma AP-550 e CP-55.

Cordaitina triangularis (auct. non) (Mehta) Hart 1965 in Marques-Toigo \& Pons, 1974, est. 2, fig. 2.

Plicatipollenites sp. cf. P. indicus (auct. non) Lele 1964 in Kemp, 1975, est. 29/I, fig. 31.

Cordaitina triangularis (auct. non) (Mehta) Hart 1965 in Ybert,1975:195, est. 4, figs. 85 e 86.

Plicatipollenites indicus (auct. non) Lele 1964 in Bharadwaj et al., 1976:75, est. 4, fig. 49.

Cordaitina sp. in Dellazzana, 1976:6-7, est.V, fig. 2 e 3.

Plicatipollenites sp. in Lima et al., 1983, est. IV, fig. 4.

Localidade tipo. Linhito Cannanore, Praia Cannanore, Costa Malabar, Terciário da Índia (Potonié \& Sah, 1960). Trata-se de espécie descrita a partir de sedimentos do Permiano redepositados no Terciário.

Diagnose. Potonié \& Sah (1960:128).

Descrição. Grão de pólen monossacado, de simetria radial e contorno circular a subcircular. Saco de margem lisa ou levemente ondulada; intramicroreticulado. Corpo central distinto, circular a subcircular. Saco inserido equatorialmente na face proximal e subequatorialmente na face distal; nesta última, com aderência associada a um sistema de pregas periférico e contínuo, acompanhando o contorno geral do grão. 
Dimensões (em 130 espécimes). Diâmetro equatorial total: 65(110)200 $\mu \mathrm{m}$; diâmetro do corpo central: 44(80)125 $\mu \mathrm{m}$; largura do saco: $8(18) 30 \mu \mathrm{m}$; recobrimento do saco no corpo central: 2,5(6) $15 \mu \mathrm{m}$.

Comparações. O sistema de pregas periférico e contínuo e a largura relativa do saco (área sem recobrimento $<2 / 3$ do raio do corpo central) constituem, de forma conjunta, os caracteres morfológicos que distinguem esta espécie das demais do gênero. Distribuição estratigráfica na bacia do Paraná. Subgrupo Itararé: Daemon \& Quadros, 1970; Kemp, 1975; Pons, 1975:38, est. III, fig. 30 e 31; Bharadwaj et al., 1976; Andreis et al., 1979, est. 3, fig. 14, 15 e 18; Dias-Fabrício, 1983, est. II, fig. 2; Lima et al., 1983; Picarelli, 1986:41, est. 3, fig. 2 e 4; Marques-Toigo, 1988:156-157, est. V, fig. 5; Marques-Toigo et al., 1989:128, est. II, fig. 12; Dias, 1993, est. 3, fig. 11; Weschenfelder et al., 1994, est. I, fig. 14; Souza, 1996:90, est, 6, fig. 5 e 6; Souza et al., 1997 , est. II, fig. 3; Souza, 2000:88, est. VII, fig. 6 e 8; Souza et al., 2000, est. 1, fig. H; Callegari, 2001:25, est. III, fig. A; Longhim et al., 2002, est. 3, fig. 4; Di Pasquo et al., 2003:301, est. 1, fig. B; Longhim, 2003:76, est. III, fig. 4; Souza et al., 2003, est. 4, fig. 3; Souza, 2003:60-62, est. 4, fig. C; Souza \& Callegari, 2004, est. 1, fig. 17; Mune, 2005, est. XIII, fig. 1. Formação Rio Bonito: Daemon \& Quadros, 1970; Ybert, 1975; Dias-Fabrício et al., 1980, est. II, fig. 1; Dias-Fabrício, 1981, est. 7, fig. 5; CazzuloKlepzig et al., 1982, est. I, fig. 13; Marques-Toigo \& Corrêa da Silva, 1984, est. 1, fig. 18; Picarelli, 1986; Marques-Toigo, 1988; Meyer, 1999:44, est. IV, fig. 4; Meyer \& Marques-Toigo, 2000, est. I, fig. 13; Souza, 2000. Formação Palermo: Cauduro, 1970; Daemon \& Quadros,1970; Leipnitz, 1981, est. V, fig. 1; CazzuloKlepzig et al., 1982; Picarelli, 1986:41, est. 3, fig. 2 e 4; MarquesToigo, 1988. Formação Irati: Bharadwaj et al., 1976; Dellazzana, 1976; Burjack, 1984, est.1, figs. 3 e 4; Picarelli, 1986; MarquesToigo, 1988.

Observações. Em Arai (1980), são ilustrados desenhos esquemáticos de grãos de pólen. Destes, aqueles das figuras 7.3 e 7.9 são relativos a Plicatipollenites malabarensis, embora o primeiro tenha sido atribuído a Cordaitina.

Plicatipollenites gondwanensis (Balme \& Hennely) Lele 1964 (Figuras 3C, D)

Nuskoisporites gondwanensis Balme \& Hennely, 1956:253 (basiônimo), est. 7, figs. 66-67.

Plicatipollenites gondwanensis (Balme \& Hennely) Lele, 1964:154-156, est. 2, fig. 11, text-fig. 4a-c e 12b.

Parasaccites gondwanensis (Balme \& Hennely) Segroves, 1969:183-186, est. 2, fig. B.

Plicatipollenites paranaensis Cauduro, 1970:14, pars, est. IX, figs. 67-69, est. X, fig. 70.

Parasaccites gondwanensis (auct. non) (Balme \& Hennely) Segroves 1969 in Menéndez, 1976:4-5, est. I, fig. 7.

Localidade tipo. Camada Big Ben, Bloomfield Colliery, Permiano da Austrália (Balme \& Hennely, 1956).

Diagnose. Lele (1964:154).

Descrição. Grão de pólen monossacado, de simetria radial e contorno circular a subcircular. Saco de margem lisa ou levemente ondulada; intramicroreticulado. Corpo central distinto, circular a subcircular. Saco inserido equatorialmente na face proximal e, subequatorialmente na face distal; nesta última, com aderência associada a um sistema de pregas poligonais relativamente afastado da periferia do corpo central. A largura do saco é igual ou maior que $1 / 2$ do raio do corpo central.

Dimensões (em 13 espécimes). Diâmetro equatorial total:76$180 \mu \mathrm{m}$; diâmetro do corpo central: 46-107 $\mu \mathrm{m}$; largura do saco: 11-45 $\mu \mathrm{m}$; recobrimento do saco no corpo central: 5(7,5)20 $\mu \mathrm{m}$. Comparações. O sistema de pregas poligonais, seu afastamento da periferia do corpo central e a largura relativa do saco diferenciam $P$. gondwanensis das outras espécies do gênero. Distribuição estratigráfica na bacia do Paraná. Subgrupo Itararé: Daemon \& Quadros, 1970:382, est. 7, forma A:P-906 e B:P-906; Dias, 1993, est. 3, fig. 3; Souza, 1996:92, est. 6, fig. 8; Souza, 2000:89, est. VIII, fig. 1; Di Pasquo et al., 2003:300, est. 1, fig. C; Formação Rio Bonito: Picarelli et al., 1987, est. 3, fig.5; Guerra-Sommer et al., 1995b, fig. 3m; Formação Palermo: Cauduro, 1970; Formação Irati: Burjack, 1984:54-55, est. I,fig. 5.

\section{Plicatipollenites trigonalis Lele 1964}

(Figuras 2E, F)

Plicatipollenites trigonalis Lele, 1964:156-157est. 2, figs. 13 e 14 , text-fig. 6a-c e $14 \mathrm{~d}$.

Plicatipollenites sp. in Daemon \& Quadros, 1970:382, est. 1, forma P-490.

Potonieisporites neglectus (auct. non) Potonié \& Lele 1961 in Lima et al., 1983 est. IV, fig. 7.

Plicatipollenites indicus (auct. non) Lele 1964 in Souza et al., 1993, est. 3, fig. 6.

Localidade tipo. Bacia South Rewa, Permiano inferior da Índia, Estágio Talchir (Lele, 1964).

Diagnose. Lele (1964, p. 156).

Descrição. Grão de pólen monossacado, de simetria radial e contorno circular a subtriangular. Saco de margem lisa ou levemente ondulada; intramicroreticulado. Corpo central distinto triangular a subcircular. Saco inserido equatorialmente na face proximal e subequatorialmente na face distal, onde está associado a um sistema de pregas de arranjo triangular, geralmente em número de três ou quatro.

Dimensões (em 24 espécimes). Diâmetro equatorial total: 80(110) $160 \mu \mathrm{m}$; diâmetro do corpo central: $45(70) 92,5 \mu \mathrm{m}$; largura do saco: $5(18) 40 \mu \mathrm{m}$; recobrimento do saco no corpo central: 5$18 \mu \mathrm{m}$.

Comparações. O arranjo triangular do sistema de pregas e a forma do corpo central diferenciam esta espécie das demais do gênero.

Distribuição estratigráfica na bacia do Paraná. Subgrupo Itararé: Daemon \& Quadros, 1970; Lima et al., 1983; Dias, 1993, est. 2, fig. 24; Souza et al., 1993; Souza, 2000:90, est. VII, fig. 7; Callegari, 2001:25, est. III, fig. B; Longhim et al., 2002, est. III, fig. 5; Longhim, 2003:77, est. III, fig. 5; Di Pasquo et al., 2003:301, est. 1, fig. A; Souza et al., 2003, est. 4, fig. 8; Souza \& Callegari, 2004, est. 1, fig. 16. Formação Rio Bonito: Dias-Fabrício, 1981, est. 7, fig. 6; Meyer, 1999:44, est. IV, fig. 6. Formação Irati: lâmina MP-P 5110, topo do Membro Assistência, no Estado de Goiás.

Plicatipollenites densus Srivastava 1970 (Figuras 3A, B) 
Plicatipollenites densus Srivastava, 1970, est. 1, fig. 7, p. 159-160. Plicatipollenites paranaensis Cauduro, 1970:14 (pars), est. X, fig. 71.

Virkkipollenites methae (auct. non) Lele 1964 in Cauduro, 1970:15, est. X, fig. 74.

Plicatipollenites indicus (auct. non) Lele 1964 in Lima et al., 1983, est. IV, fig. 1.

Localidade tipo. Dera Colliery, Carvão Talchir, Permiano inferior da Índia, Estágio Barakar (Srivastava, 1970).

Diagnose. Srivastava (1970, p. 160).

Descrição. Grão de pólen monossacado, de simetria radial e contorno circular a subcircular. Saco de margem lisa ou levemente ondulada; intramicroreticulado. Corpo central distinto, circular a subcircular. Saco inserido equatorialmente na face proximal e subequatorialmente na face distal, onde é associado a um sistema de pregas contínuo, periférico. A largura do saco, na área sem recobrimento, atinge, no mínimo, $2 / 3$ do raio do corpo central.

Dimensões (em 20 espécimes). Diâmetro equatorial total: 77,5(110) $130 \mu \mathrm{m}$; diâmetro do corpo central: $40(55) 70 \mu \mathrm{m}$; largura do saco: $18-30 \mu \mathrm{m}$; recobrimento do saco no corpo central: 2,5(5)12,5 $\mu \mathrm{m}$.

Comparações. O sistema de pregas contínuo e periférico distingue essa espécie de $P$. gondwanensis e $P$. trigonalis e a largura relativa do saco a distingue de $P$. malabarensis. $\mathrm{Na}$ diagnose da espécie (Lele, 1964, p. 156) é demonstrado que o corpo central é somente "ocasionalmente" mais denso que o saco, razão pela qual este caráter não deve ser adotado para distinção entre as espécies do gênero. Neste trabalho adotase a proposição de Azcuy \& Gutiérrez (1985), pela qual são alocados em $P$. densus os espécimes cujos sacos têm largura, na área livre, maior que $2 / 3$ do raio do corpo central.

Distribuição estratigráfica na bacia do Paraná. Subgrupo Itararé: Lima et al., 1983; Dias, 1993, est. 3, fig. 4; Souza, 1996:92, est. 6, fig. 3 e 7; Souza, 2000:91, est. VIII, fig. 2; Longhim et al., 2002, est. III, fig. 7; Longhim, 2003:78, est. III, fig. 7; Souza et al., 2003:62, est. 4, fig. 4; Mune, 2005, est. XIII, fig. 2. Formação Rio Bonito: Meyer, 1999:43, est. IV, fig. 5; Souza, 2000. Formação Palermo: Cauduro, 1970.

Observações. O espécime atribuído a Cannanoropollis methae por Bharadwaj et al. (1976, est. 4, fig. 50), com ocorrência nas bacias do Paraná e Parnaíba, apresenta sistema de pregas associado à aderência do saco no corpo central e dimensões da largura do saco que permitem supor tratar-se de Plicatipollenites densus. No entanto, uma designação mais segura é limitada pela ausência de descrição no trabalho original.

\section{Infraturma VESICULOMONORADITES Pant 1954 Crucisaccites Lele \& Maithy 1964}

Espécie-tipo. Crucisaccites latisulcatus Lele \& Maithy 1964. Afinidade botânica. Gymnospermopsida (Gutiérrez, 1993). Aspectos gerais. O gênero inclui grãos de pólen monossacados, de simetria bilateral e contorno circular a oval; o corpo central acompanha o contorno geral do grão. Saco intramicroreticulado, com inserção cruzada no corpo central, distal e proximalmente, encobrindo a maior parte deste. Em ambos os lados, o saco apresenta uma zona bilateral em formato de sulco alongado, de largura variável; saco com ou sem sistema de pregas de aderência ao corpo central.

Comparação com outros gêneros. Este táxon diferencia-se dos demais gêneros de monossacados devido à presença de sulco nas duas faces do saco, que se arranjam ortogonalmente.

\section{Crucisaccites latisulcatus Lele \& Maithy 1964}

(Figuras 3C, D)

Crucisaccites latisulcatus Lele \& Maithy, 1964, est. 1, figs. 16, text-fig. 2a-fe 2g-i.

Localidade tipo. Central Pit, Serampur Colliery, Carvão Giridith, Permiano inferior da Índia, Estágio Karharbari (Lele \& Maithy, 1964).

Diagnose. Lele \& Maithy (1964:309).

Descrição. Grão de pólen monossacado, de simetria bilateral e contorno circular a oval; o corpo central acompanha o contorno geral do grão. Saco intramicroreticulado com inserção cruzada no corpo central, distal e proximalmente, encobrindo a maior parte deste. Em ambos os lados, o saco apresenta uma zona bilateral em formato de sulco alongado e de largura variável; saco com sistema de pregas de aderência ao corpo central.

Dimensões (em 3 espécimes). Diâmetro equatorial total: 111$125 \mu \mathrm{m}$; diâmetro do corpo central: 80-110 $\mu \mathrm{m}$.

Distribuição estratigráfica na bacia do Paraná. Subgrupo Itararé: Sundaram, 1986, est. VII, fig. 6; Souza, 2000:91, est. VIII, fig. 3 e 4; Souza, 2003, fig. 4E.

\section{Crucisaccites monoletus Maithy 1965}

(Figuras 3E, F)

Crucisaccites monoletus Maithy, 1965 :295, est. 3, fig. 6.

Localidade tipo. Carvão Giridith, Bihar, Permiano inferior da Índia (Maithy, 1965).

Diagnose. Maithy (1965:295).

Descrição. Grão de pólen monossacado, de simetria bilateral e contorno circular a oval; o corpo central acompanha o contorno geral do grão. Saco intramicroreticulado com inserção cruzada no corpo central, distal e proximalmente, encobrindo a maior parte deste. Em ambos os lados, o saco apresenta uma zona bilateral em formato de sulco alongado e de largura variável; saco sem sistema de pregas de aderência ao corpo central.

Dimensões (em 6 espécimes). Diâmetro equatorial total: 87,5$128 \mu \mathrm{m}$; diâmetro do corpo central: $95-90 \mu \mathrm{m}$.

Distribuição estratigráfica na bacia do Paraná. Subgrupo Itararé: Souza, 2000:92, est. VIII, fig. 5; Souza et al., 2000, est. I, fig 1; Longhim et al., 2002 :51, est. III, fig. 8; Longhim, 2003:79, est. III, fig. 8; Di Pasquo et al., 2003:300, fig. 2A; Souza \& Marques-Toigo, 2003, fig. 3D.

Observações. A análise do espécime registrado por Pons (1975, est. 5, fig. 39, p. 43) na seção superior do Subgrupo Itararé em Santa Catarina, atribuído a Crucisaccites cf. Crucisaccites monoletus, é dificultada pela ausência de suas dimensões e qualidade da fotomicrografia. 


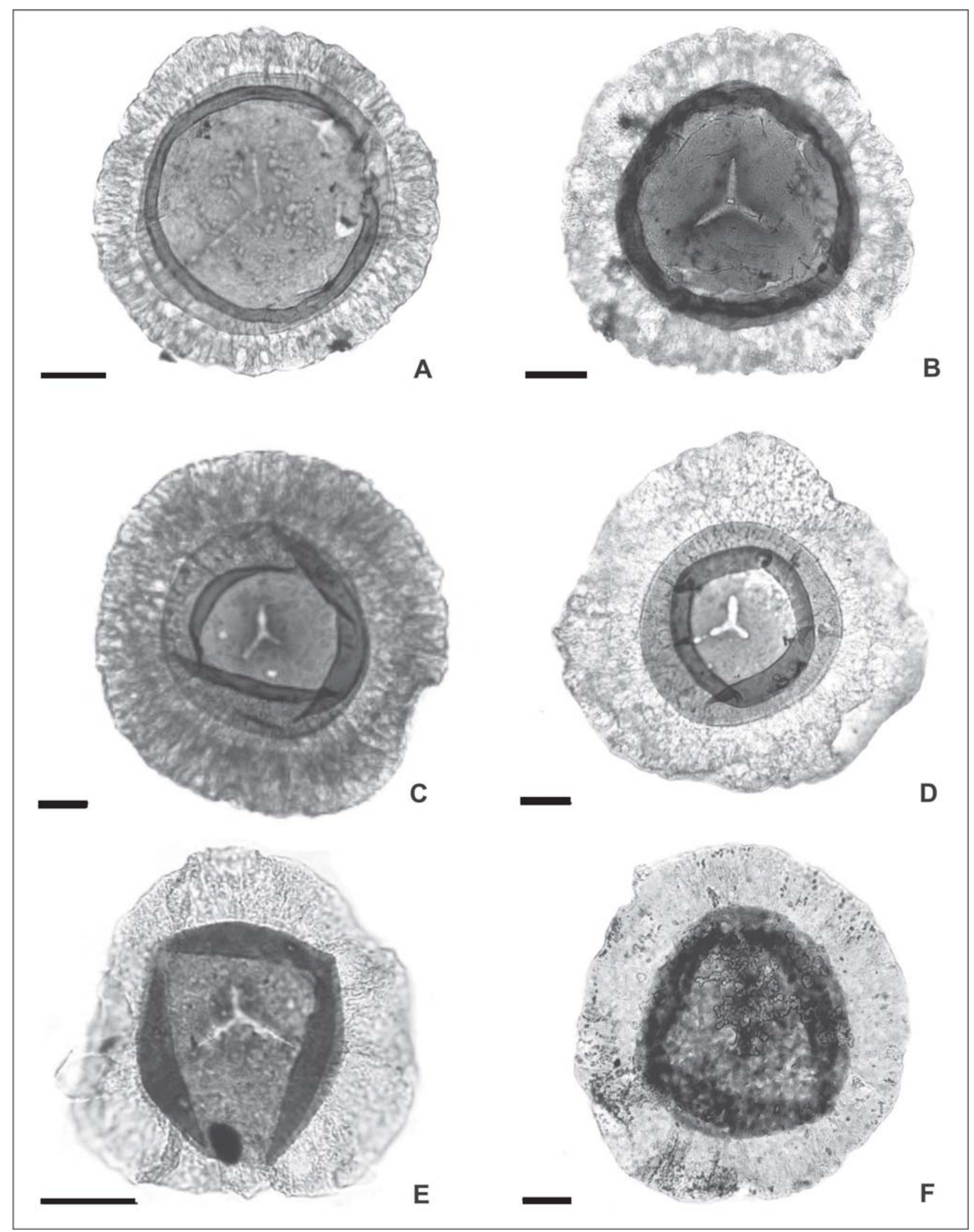

Figura 2. Fotomicrografias de Plicatipollenites (entre parênteses o número da lâmina e as coordenadas England Finder): A, $P$. malabarensis (MP-P 28, S35); B, P. malabarensis (MP-P 46, M40). C, P. gondwanensis (MP-P 55, J40); D, P. gondwanensis (MP-P 55, N40). E, P. trigonalis (GP4E 1386, U40); F, P. trigonalis (MP-P 5072, M33). Escala $=20 \mu \mathrm{m}$.

Figure 2. Plicatipollenites photomicrographs (in parenthesis are the slide number and the England Finder coordinates). Scale bar $=20 \mu \mathrm{m}$. 


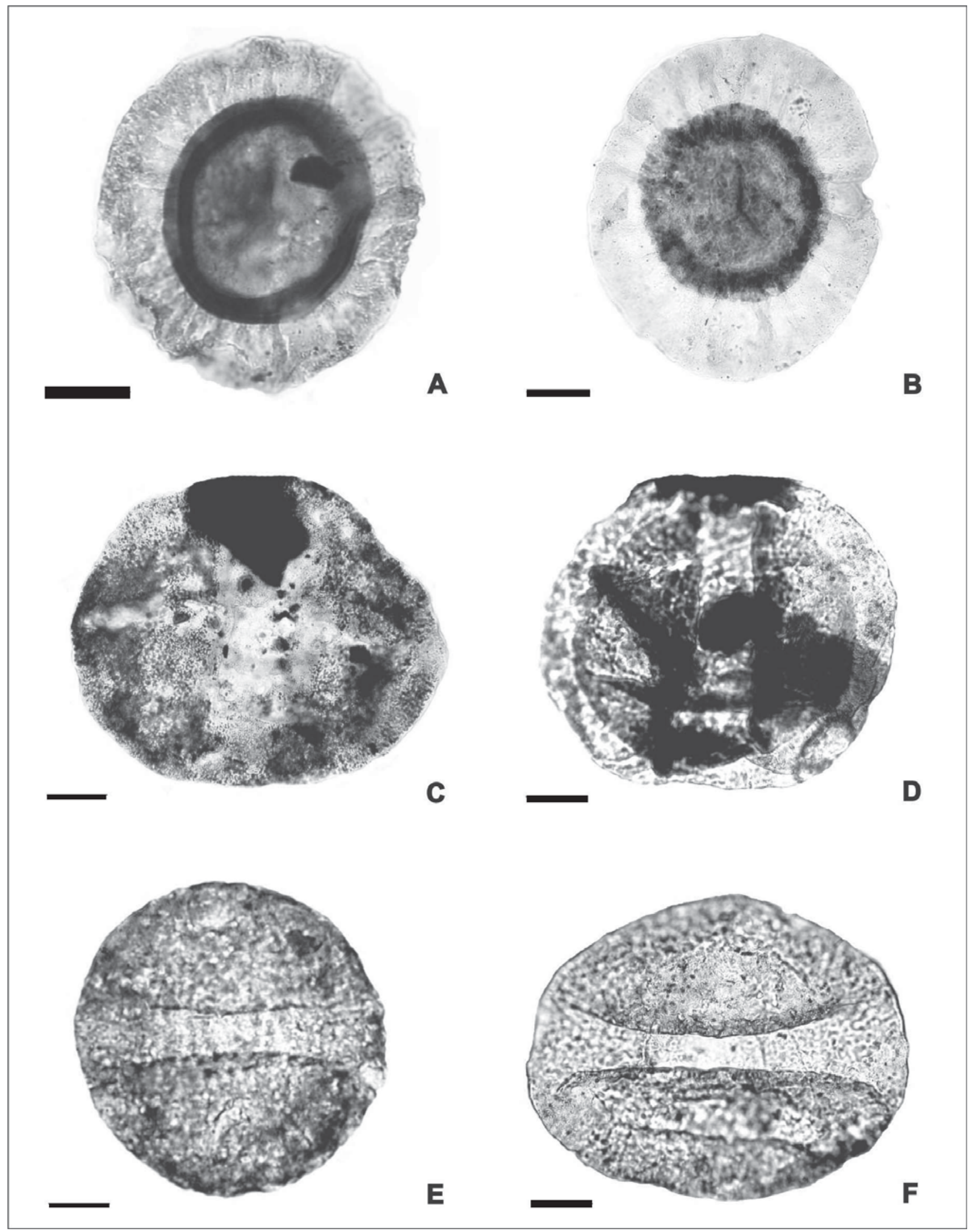

Figura 3. Fotomicrografias de Plicatipollenites e Crucisaccites (entre parênteses o número da lâmina e as coordenadas England Finder): A, P. densus (GP4E 1419, U53-3); B, P. densus (MP-P 5074, 031-3); C, C. latisulcatus (IG-P 13-B, Q57); D, C. latisulcatus (MP-P 5075, D4); E, C. monoletus (MP-P 5073, J32-1); F, C. monoletus (MP-P 5074, J42). Escala $=20 \mu \mathrm{m}$.

Figure 3. Plicatipollenites and Crucisaccites photomicrographs (in parenthesis are the slide number and the England Finder coordinates). Scale bar $=20 \mu \mathrm{m}$. 


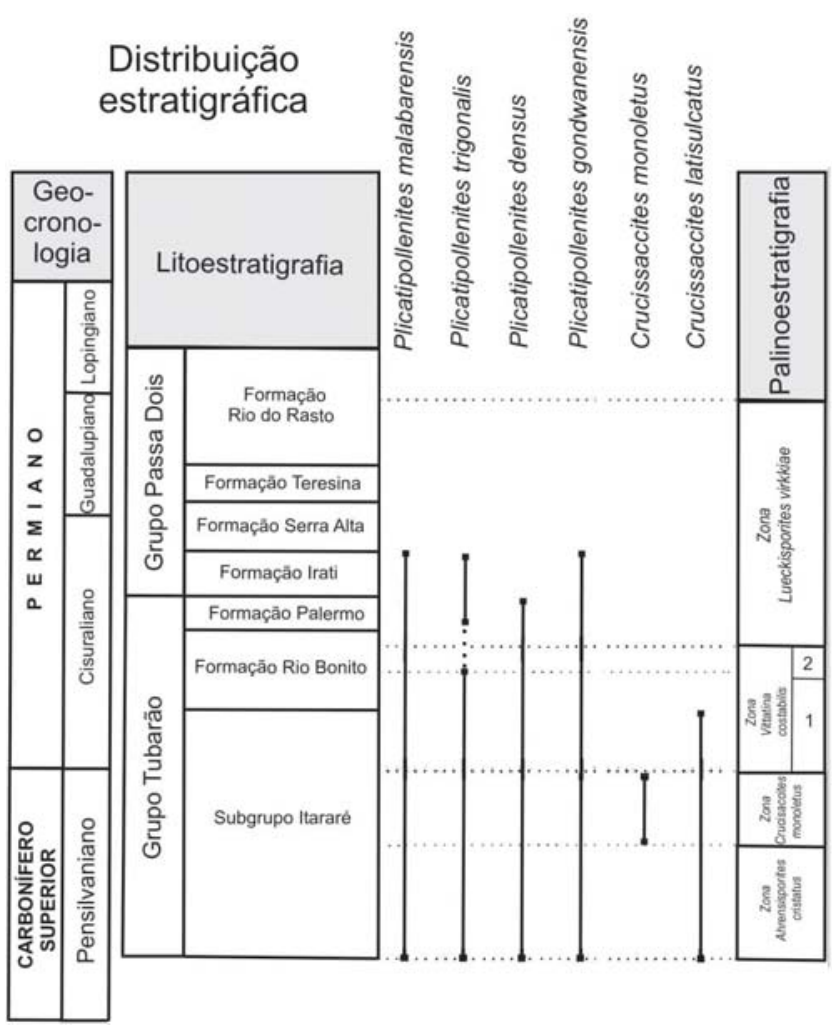

Figura 4. Amplitude lito e palinobioestratigráfica das espécies de Plicatipollenites e Crucisaccites na bacia do Paraná: 1, Subzona Protohaploxypinus goraiensis; 2, Subzona Hamiapollenites karroensis (segundo Souza \& Marques-Toigo, 2005).

Figure 4. Litostratigraphic and palynobiostratigraphic ranges of Plicatipollenites and Crucisaccites species in Paraná Basin: 1, Subzone Protohaploxypinus goraiensis; 2, Subzone Hamiapollenites karroensis (according to Souza \& Marques-Toigo, 2005).

\section{CONSIDERAÇÕES FINAIS}

Grãos de pólen monossacados de simetria radial e bilateral são relativamente abundantes em estratos do Carbonífero Superior ao Permiano Médio do Gondwana, com destaque aos gêneros Plicatipollenites e Cannanoropollis, que ocorrem com significativa variação morfológica, justificando o grande número de espécies. Contrariamente, o gênero Crucisaccites é quantitativamente pouco expressivo, ressaltando-se somente duas espécies (C. latisulcatus e C. monoletus). Estas constatações são verificadas, praticamente em todas as áreas gondvânicas (ver Foster, 1975, 1979; Gutiérrez, 1993; Lindström, 1995; Azcuy \& Di Pasquo, 2000). Contudo, a distribuição estratigráfica dessas espécies varia de bacia para bacia, controlada, essencialmente, por provincialismos de natureza paleoclimática.

A amplitude estratigráfica das seis espécies abordadas neste trabalho é apresentada na Figura 4, cuja geocronologia é baseada em dados radiométricos inéditos (Santos et al., no prelo) e correlações com o Gondwana Ocidental (Souza \& Marques-Toigo, 2003, 2005; Iannuzzi \& Souza, 2005). A análise da distribuição das espécies dos dois gêneros na bacia do Paraná permite, em primeira instância, a confirmação de uma espécie de valor bioestratigráfico, Crucisaccites monoletus, restrita à porção média do Subgrupo Itararé. Além disso, alguns problemas taxonômicos são ajustados, a exemplo dos espécimes atribuídos à nova espécie Plicatipollenites paranaensis proposta por Cauduro (1970), cuja rejeição já havia sido preconizada por outros autores (Gutiérrez, 1993; Premaor et al., 2004). Entretanto, nem todos os espécimes a ele consignados são relativos a Plicatipollenites gondwanensis, conforme atestam as listas sinonímicas aqui apresentadas. Essas observações demonstram a importância de estudos taxonômicos detalhados numa das seções que é, comprovadamente, uma das mais espessas do Paleozóico Superior do Gondwana, com fortes implicações de caráter bioestratigráfico.

Uma melhor análise taxonômica ainda seria possível se houvesse disposição de uma base de dados qualitativa e quantitativamente mais confiável, em razão de que, principalmente, vários trabalhos de circulação restrita não foram devidamente publicados (e.g., Leipnitz, 1981; Burjack, 1984; Sundaram, 1986). Além disso, encoraja-se a realização de descrições taxonômicas em cada trabalho, de modo a facilitar análises ulteriores e o uso de ilustrações originais para cada localidade.

\section{AGRADECIMENTOS}

Agradecimentos são feitos à PETROBRAS, FAPERGS e ao CNPq pela concessão de bolsas e pelo auxílio a projetos de pesquisa (FAPERGS 02/1755-2; CNPq 303368/2004-7 e 474153/ 20045).

\section{REFERÊNCIAS}

Andreis, R.R.; Cazzulo-Klepzig, M.; Guerra-Sommer, M. \& Marques-Toigo, M. 1979. Interpretação paleoambiental e estudo paleobotânico e palinológico do Grupo Itararé, na área de Faxinal, município de Guaíba. In: SIMPÓSIO DE GEOLOGIA DO NORDESTE, 9, 1979. Atas, Natal, p. 65-77.

Arai, M. 1980. Contribuição dos pólens estriados na bioestratigrafia neopaleozóica da parte NE da bacia do Paraná. Boletim IGUSP, 11:53-64.

Azcuy, C.L. \& Di Pasquo, M. 2000. Palynology of the Late Carboniferous from the Tarija Basin, Argentina: a systematic rewiew of the monosaccate pollen genera. Palaeontographica, Abt. B253:7-37.

Azcuy, C.L. \& Gutiérrez, P.R. 1985. Palinología de sedimentitas carbónicas de la Cuenca San Rafael. Ameghiniana, 22(1-2):97-109.

Balme, B.E. \& Hennelly, J.P.F. 1956. Trilete sporomorphs from Australian Permian sediments. Australian Journal of Botany, 4(3):240-60.

Bharadwaj, D.C. 1957. The Palynological investigations of the Saar coals. Paleontographica, Abt. B101:73-125.

Bharadwaj, D.C.; Kar, R.K. \& Navale, G.K.B. 1976. Palynostratigraphy of Lower Gondwana deposits in Paraná and Maranhão Basins, Brazil. Biological Memoirs, 1(1-2):56-103.

Bose, M.N. \& Kar, R.K. 1966. Paleozoic sporae dispersae from Congo I. Kindu-Kalima and Walikale regions. Annales du Musee Royal de L'Afrique Centrale Serie IN-8 ${ }^{\circ}$, 53:1-250.

Burjack, M.I.A. 1984. Caracterização da matéria orgânica dispersa nos sedimentitos da Formação Irati, Permiano Superior da bacia do Paraná. Programa de Pós-graduação em Geociências, 
Universidade Federal do Rio Grande do Sul, Tese de Doutoramento, $231 \mathrm{p}$.

Callegari, L. M. 2001. Palinologia da porção superior do Subgrupo Itararé na região de Jumirim e Tietê, Estado de São Paulo. Instituto de Geociências, Universidade de São Paulo, Monografia, $52 \mathrm{p}$.

Cauduro, A.D. 1970. Lower Gondwana miospores from São Sepé outcrop (Rio Grande do Sul, Brasil). Escola de Geologia da UFRGS, Publicação Especial 17, 34 p.

Cazzulo-Klepzig, M.; Dias-Fabrício, M.E. \& Marques-Toigo, M. 1982. Palinological characterization of rocks associated to coal seams of Santa Rita coalfield, Rio Bonito and Palermo Formations, Paraná Basin, Permian, Rio Grande do Sul, Brazil. In: CONGRESSO GEOLÓGICO CHILENO, 3, 1982. Actas, Concepción, p. 65-83.

Clement-Westerhoff, J.A. 1984. Aspects of Permian Palaeobotany and Palynology. IV. The Conifer Ortiseia Florin from the Val Gardena Formation on the Dolomites and the Vicentinian Alps (Italy) with special reference to a revised concepts of the Walchiaceae (Göppert) Schimper. Review of Palaeobotany and Palynology, 41(1-2):51-166.

Daemon, R.F. \& Quadros, L.P. 1970. Bioestratigrafia do Neopaleozóico da bacia do Paraná. In: CONGRESSO BRASILEIRO DE GEOLOGIA, 24, 1970. Anais, Brasília, p. 359-412.

Dellazzana, J.G. 1976. Contribuição à palinologia da Formação Irati (Permiano) Rio Grande do Sul, Brasil. Ameghiniana, 13(1):1-42.

Di Pasquo, M.; Azcuy, C.L.; Souza, P.A. 2003. Palinología del Carbonífero Superior del Subgrupo Itararé en Itaporanga, Cuenca Paraná, Estado de São Paulo, Brasil. Parte 2: sistemática de pollen y significado paleoambiental y estratigráfico. Ameghiniana, 40(3):297-313.

Dias, M.E. 1993. Palinologia do Grupo Itararé na porção centrosul do Rio Grande do Sul, Permiano da bacia do Paraná, Brasil. Pesquisas, 20(2):119-131.

Dias-Fabrício, M.E. 1981. Palinologia da Formação Rio Bonito na área de Gravataí-Morungava, Rio Grande do Sul. Pesquisas, 14:69-130.

Dias-Fabrício, M.E. 1983. Micrósporos da bacia carbonífera de Gravataí-Morungava, Permiano da bacia do Paraná, Rio Grande do Sul, Brasil. In: SIMPÓSIO SUL-BRASILEIRO DE GEOLOGIA, 1, 1983. Atas, Porto Alegre, p. 170-183.

Dias-Fabrício, M.A.; Piccoli, A.E.M. \& Marques-Toigo, M. 1980. Interpretação paleogeográfica com base em dados palinológicos da Formação Rio Bonito (Pemiano Inferior), bacia do Paraná, RS. In: CONGRESSO BRASILEIRO DE GEOLOGIA, 31, 1980. Anais, Balneário Camboriú, v. 2, p. 729-739.

Foster, C.B. 1975. Permian plant microfossils from the Blair Athol Coal Measures, Central Queensland, Australia. Palaeontographica, Abt. B154:121-171.

Foster, C.B. 1979. Permian plant microfossils of the Blair Athol Coal Measures, Baralaba Coal Measures, and Basal Rewan Formation of Queensland. Geological Survey of Queensland, 372(45):1-154.

Guerra-Sommer, M.; Cazzulo-Kleipzig, M. \& Marques-Toigo, M. 1995a. Paleoclimatic implications of Lycophyta in the Gondwana of Southern Brazil. Pesquisas, 22(1-2):21-31.

Guerra-Sommer, M.; Cazzulo-Kleipzig, M. \& Marques-Toigo, M. 1995b. Gondwanostachyaceae (Equisetopsida) no Gondwana sul-brasileiro (Formação Rio Bonito) com mega e microflora associadas. Pesquisas, 22(1-2):64-73.

Gutiérrez, P.R. 1993. Palinologia de la Formación Agua Colorada
(Carbonífero Superior), Sierra de Famatina, Provincia de La Rioja, Argentina. I. Granos de polen. Ameghiniana, 30(2):163-212.

Iannuzzi, R. \& Souza, P.A. 2005. Floral sucession in the Lower Permian deposits of the Brazilian Paraná Basin: an up-to-dated overview. New Mexico Museum of the Natural History and Science Bulletin, 30:144-149.

Jansonius, J. \& Hills, L.V. 1976. Genera file of fossil spore. Special Publication of Department Geology, Univesity of Calgary, Canadá.

Kemp, E.M. 1975. The palynology of Late Palaeozoic glacial deposits of Gondwanaland. In: GONDWANA SYMPOSIUM, 3, 1975. Artigo, Canberra, Australian National University, p. 397-413.

Leipnitz, B. 1981. Estudo palinológico da Formação Palermo, Estado de Santa Catarina, Permiano, bacia do Paraná, Brasil. Programa de Pós-Graduação em Geociências, Universidade Federal do Rio Grande do Sul, Dissertação de Mestrado, $141 \mathrm{p}$.

Lele, K.M. 1964. Studies in the Talchir Flora of India: 2.Resolution of the spore genus Nuskoisporites Pot. \& K.L. The Palaeobotanist, 12(2):146-170.

Lele, K.M. \& Maithy, P.K. 1964. An unusual monosaccate spore from the Karharbari stage, Giridih coalfield, India. The Paleobotanist, 12(3):307-313.

Lima, M.R.; Dino, R. \& Yokoya, N.S. 1983. Palinologia de concreções calcíferas do Subgrupo Itararé (Neopaleozóico da bacia do Paraná) da região de Araçoiaba da Serra, Estado de São Paulo. Anais da Academia Brasileira de Ciências, 55(2):195-208.

Lindström, S. 1995. Early Permian palynostratigraphy of the northern Heimefrontfjella mountain-range, Dronning Maud Land, Antarctica. Review of Palaeobotany and Palynology, 89:359-415.

Longhim, M.E. 2003. Palinologia do Grupo Itararé em Salto, Estado de São Paulo (bacia do Paraná, Carbonífero Superior). Programa de Pós-graduação em Geologia, Universidade Estadual Paulista, Dissertação de Mestrado, 127 p.

Longhim, M.E.; Souza, P.A. \& Rohn, R. 2002. Palinologia do Grupo Itararé na região de Salto (Carbonífero Superior), Estado de São Paulo, Brasil. Parte 1- Palinologia Sistemática. Revista Universidade Guarulhos, Geociências, VII(6):43-60.

Maithy, P.K. 1965. Studies in the Glossopteris flora of India. 27. Sporae dispersae from the Karharbari beds in the Giridish coalfield, Bihar. The Palaeobotanist, 13(3):291-307.

Marques-Toigo, M. 1988. Palinologia, bioestratigrafia e paleoecologia do Neopaleozóico da bacia do Paraná nos estados do Rio Grande do Sul e Santa Catarina, Brasil. Programa de Pós-Graduação em Geociências, Universidade Federal do Rio Grande do Sul, Tese de Doutoramento, 259 p.

Marques-Toigo, M. \& Corrêa da Silva, Z.C. 1984. On the origin of gondwanic South Brazilian Coal Measures. Comunicações do Serviço Geológico de Portugal, 70(2):151-160.

Marques-Toigo, M.; Dias-Fabrício, M.E.; Guerra-Sommer, M.; CazzuloKlepzig, M. \& Piccoli, A.E.M. 1989. Afloramentos da área de Trombudo Central, Permiano Inferior, Santa Catarina: palinologia, icnologia e sedimentologia. In: CONGRESSO BRASILEIRO DE PALEONTOLOGIA, 11, 1989. Anais, Curitiba, p. 125-150.

Marques-Toigo, M. \& Pons, M.E. 1974. Estudo palinológico do furo de sondagem P7 malha oeste da bacia carbonífera do Iruí, RS- Brasil. In: CONGRESSO BRASILEIRO DE GEOLOGIA, 28, 1974. Anais, Porto Alegre, p. 503-515.

Menéndez, C.A. 1976. Contenido palinológico de estratos pérmicos con "Mesosaurus" de Rio Claro, São Paulo, Brasil. Revista Del Museu Argentino de Ciências Naturales "Bernardino Rivadavia”, II(1):1-30. 
Meyer, K. 1999. Caracterizacão palinológica das camadas de carvão da Malha IV na Mina de Candiota, RS, Permiano da bacia do Paraná. Programa de Pós-graduação em Geociências, Universidade Federal do Rio Grande do Sul, Dissertação de Mestrado, 112 p.

Meyer, K.E.B. \& Marques-Toigo, M. 2000. O significado paleoambiental da microflora dos carvões da malha IV, mina de Candiota, RS, Permiano da bacia do Paraná, Brasil. Revista Universidade Guarulhos, Geociências, V(volume especial):17-20.

Milani, E.J. \& Zalán, P.V. 1999. An outline of the geology and petroleum systems of the Paleozoic interior basins of South America. Episodes, 22:199-205.

Milani, J.E. 1997. Evolução tectono-estratigráfica da Bacia do Paraná e seu relacionamento com a geodinâmica fanerozóica do Gondwana sul-oriental. Programa de Pós-graduação em Geociências, Universidade Federal do Rio Grande do Sul, Tese de Doutoramento, 255 p.

Mune, S.E. 2005. Tafoflora interglacial neocarbonifera do Sítio Volpe, Município de Monte Mor (SP), Subgrupo Itararé, nordeste da bacia do Paraná: revisão e complementação. Programa de Pós-graduação em Geologia Sedimentar, Universidade de São Paulo, Dissertação de Mestrado, 137 p.

Picarelli, A.T. 1986. Palinologia dos testemunhos de sondagem 2TG-69-RS e 2-TG-99-RS da jazida carbonifera de Santa Terezinha, RS, Brasil-Permiano da bacia do Paraná. Programa de Pós-graduação em Geociências, Universidade Federal do Rio Grande do Sul, Dissertação de Mestrado, 137 p.

Picarelli, A.T.; Dias Fabrício, M.A. \& Cazzulo-Klepzig, M. 1987. Considerações sobre a paleoecologia e a palinologia da jazida carbonífera de Santa Terezinha, RS, Brasil - Permiano da bacia do Paraná. In: SIMPÓSIO SUL-BRASILEIRO DE GEOLOGIA, 3, 1987. Atas, Curitiba, p. 351-372.

Piccoli, A.E.M.; Menegat, R.; Guerra-Sommer, M.; Marques-Toigo, M. \& Porcher, C.C. 1991. Faciologia da seqüência sedimentar das folhas de Quitéria e Várzea do Capivarita, Rio Grande do Sul. Pesquisas, 18(1):31-43.

Playford, G. \& Dino, R. 2000. Palynostratigraphy of upper Palaeozoic strata (Tapajós Group), Amazonas Basin, Brazil: Part two. Paleontographica, Abt. B255(4-6):87-145.

Pons, M.E. 1975. Estudo palinológico do Sub-Grupo Itararé na "Coluna White”, Permiano Inferior, Santa Catarina. Programa de Pós-graduação em Geociências, Universidade Federal do Rio Grande do Sul, Dissertação de Mestrado, 104 p.

Potonié, R. 1967. New phylogenetic facts on fossil spores. Review of Palaeobotany and Palynology, 1:75-82.

Potonié, R. 1970. Synopsis der Gattungen der Sporae Dispesae. V Teil: Nachträge zur allen Gruppen (Turmae). Geologischen Jahrbuch, 87:1-172.

Potonié, R. \& Kremp, G.O.U. 1954. Die Gattungen der Paläozoischen sporae dispersae und ihre Stratigraphie. Geologischen Jahrbuch, 69:111-194.

Potonié, R. \& Sah, S.D.C. 1960. Sporae dispersae of the lignites from Cannanore Beach on the Maslaber Coast of India. The Palaeotobanist, 7(2):121-135.

Premaor, E.; Smaniottto, L.P.; Souza, J.M. \& Souza, P.A. 2004. Considerações palinotaxônomicas sobre a Formação Palermo, Permiano da bacia do Paraná. In: REUNIÃO DE PALEOBOTÂNICOS E PALINÓLOGOS, 11, 2004. Boletim de Resumos, Gramado, p. 117.

Santos, R.V.; Souza, P.A.; Alvarenga, C.J.S.; Dantas, E.L.; Pimentel, M.M.; Oliveira, C.G. \& Araújo, L.M. no prelo. Shrimp U-Pb zircon dating and palynology of bentonitic layers from the Permian Irati Formation. Gondwana Research, 9.

Segroves, K.L. 1969. Saccate plant microfossils from the Permian of Western Austrália. Grana Palynologica, 9(1-3):174-227.

Souza, P.A. 1996. Palinologia e bioestratigrafia do Subgrupo Itararé em Araçoiaba da Serra (Westphaliano, bacia do Paraná), Estado de São Paulo, Brasil. Programa de Pós Graduação em Geologia Sedimentar, Universidade de São Paulo, Dissertação de Mestrado, $192 \mathrm{p}$.

Souza, P.A. 2000. Palinobioestratigrafia do Subgrupo Itararé, Carbonífero/Permiano, na porção nordeste da bacia do Paraná (SP/PR, Brasil). Programa de Pós-graduação em Geologia Sedimentar, Universidade de São Paulo, Tese de Doutoramento, $199 \mathrm{p}$.

Souza, P.A. 2003. New Palynological data of the Itararé Subgroup from the Buri Coal (Late Carboniferous, Paraná Basin), São Paulo State, Brazil. Revista Brasileira de Paleontologia, 5:49-58.

Souza, P.A. \& Callegari, L.M. 2004. An Early Permian palynoflora from the Itararé Subgroup, Paraná Basin, Brazil. Revista Española de Micropaleontologia, 36(3):439-450.

Souza, P.A.; Batezelli, C.V.B.; Di Pasquo, M.; Azcuy, C.L.; Saad, A.R. \& Perinotto, J.A.J. 2000. Ocorrência de palinomorfos no Subgrupo Itararé (C/P da bacia do Paraná) em Jundiaí (SP, Brasil). Revista Universidade Guarulhos, Geociências, V(volume especial):28-32.

Souza, P.A.; Lima, M.R. \& Saad, A.R. 1993. Palinologia dos Carvões Paleozóicos do Estado de São Paulo, Brasil. I - O Carvão de Buri. Revista do Instituto Geológico, 14(1):5-20.

Souza, P.A., Lima, M.R. \& Saad, A.R., 1997. Palinologia dos carvões paleozóicos do Estado de São Paulo, Brasil. II - O carvão de Monte Mor. Revista do Instituto Geológico, 18(12):7-21.

Souza, P. A. \& Marques-Toigo, M. 2003. An overview on the palynostratigraphy of the Upper Paleozoic strata of the Brazilian Paraná Basin. Revista del Museo Argentino de Ciencias Naturales, nueva serie, 5(2):205-214.

Souza, P. A. \& Marques-Toigo, M. 2005. Progress on the palynostratigraphy of the Permian strata in Rio Grande do Sul State, Paraná Basin, Brazil. Anais da Academia Brasileira de Ciências, 77(2):353-365.

Souza, P.A.; Petri, S. \& Dino, R. 2003. Late Carboniferous palynology from the Itararé Subgroup (Paraná Basin) at Araçoiaba da Serra, São Paulo State, Brazil. Palynology, 27:39-74.

Srivastava, S.C., 1970. Miofloral investigations in some coals of Talchir Coalfield (Orissa) India. Lucknow, India. The Palaeobotanist, 18(2):154-166.

Sundaram, D. 1986. Palinologia do Subgrupo Itararé (Neopaleozóico), bacia do Paraná, no Estado de São Paulo, Brasil. Programa de Pós-graduação em Geologia Sedimentar, Universidade de São Paulo, Tese de Doutoramento, 311 p.

Weschenfelder, J.; Kirchheim, R.E.; Scherer, C.M.S. \& CazzuloKlepzig, M. 1994. Ocorrência de rochas sedimentares Gondwânicas Eo-Permianas sobre a borda leste do escudo sulrio-grandense. Acta Geologica Leopoldensia, 39(2):599-614.

Ybert, J.P. 1975. Etude des miospores du Basin Houiller de CandiotaHulha Negra, Rio Grande do Sul, Bresil. Pesquisas, 5:181-226.

Received in January, 2006; accepted in March, 2006. 